\title{
An induced fit mechanism regulates p53 DNA binding kinetics to confer sequence specificity
}

\author{
Tom J Petty ${ }^{1,2,8}$, Soheila Emamzadah ${ }^{1,8}$, \\ Lorenzo Costantino ${ }^{1,8}$, Irina Petkova ${ }^{3}$, \\ Elena S Stavridi ${ }^{4,7}$, Jeffery G Saven ${ }^{5}$, \\ Eric Vauthey ${ }^{3}$ and Thanos D \\ Halazonetis ${ }^{1,6, *}$
}

\begin{abstract}
${ }^{1}$ Department of Molecular Biology, University of Geneva, Geneva, Switzerland, 'enomics and Computational Biology Graduate Group, University of Pennsylvania, Philadelphia, PA, USA, ${ }^{3}$ Department of Physical Chemistry, University of Geneva, Geneva, Switzerland,

${ }^{4}$ The Wistar Institute, Philadelphia, PA, USA, ${ }^{5}$ Department of Chemistry, University of Pennsylvania, Philadelphia, PA, USA and ${ }^{6}$ Department of Biochemistry, University of Geneva, Geneva, Switzerland
\end{abstract}

The p53 tumour suppressor gene, the most frequently mutated gene in human cancer, encodes a transcription factor that contains sequence-specific DNA binding and homo-tetramerization domains. Interestingly, the affinities of $\mathbf{p 5 3}$ for specific and non-specific DNA sites differ by only one order of magnitude, making it hard to understand how this protein recognizes its specific DNA targets in vivo. We describe here the structure of a 553 polypeptide containing both the DNA binding and oligomerization domains in complex with DNA. The structure reveals that sequencespecific DNA binding proceeds via an induced fit mechanism that involves a conformational switch in loop L1 of the p53 DNA binding domain. Analysis of loop L1 mutants demonstrated that the conformational switch allows DNA binding off-rates to be regulated independently of affinities. These results may explain the universal prevalence of conformational switching in sequence-specific DNA binding proteins and suggest that proteins like p53 rely more on differences in binding off-rates, than on differences in affinities, to recognize their specific DNA sites. The EMBO Journal (2011) 30, 2167-2176. doi:10.1038/ emboj.2011.127; Published online 26 April 2011

Subject Categories: chromatin \& transcription; genome stability \& dynamics

Keywords: affinity; crystallography; induced fit; off-rate; p53

\section{Introduction}

The most frequently mutated gene in human cancer is $p 53$ (Hollstein et al, 1991; Kan et al, 2010), a gene encoding a sequence-specific DNA binding protein (Kern et al, 1991). The p53 protein is activated in response to DNA damage and

*Corresponding author. Department of Molecular Biology, University of Geneva, 30 Quai Ernest-Ansermet, Geneva 1205, Switzerland.

Tel.: + 4122379 6112; Fax: + 4122379 6868;

E-mail: thanos.halazonetis@unige.ch

${ }^{7}$ Present address: Merck-Serono, Geneva, Switzerland

${ }^{8}$ These authors contributed equally to this work

Received: 3 February 2011; accepted: 24 March 2011; published online: 26 April 2011 then enhances transcription of genes that induce cell-cycle arrest, apoptosis or senescence (Kastan et al, 1991; Kuerbitz et al, 1992; Vogelstein et al, 2000). To explain the high frequency of $p 53$ mutations in human cancer, it has been proposed that activation of oncogenes in precancerous lesions leads to DNA damage, which in turn curtails, in a p53-dependent manner, the macroscopic growth of the lesion (Bartkova et al, 2005; Gorgoulis et al, 2005). According to this model, inactivation of p53 is an important step in the progression of precancerous lesions to cancer, because it allows cancer cells to proliferate despite the presence of oncogene-induced DNA damage (Halazonetis et al, 2008).

Given the central role of the p53 protein in human cancer, it is not surprising that significant effort has been devoted towards elucidating its function and structure at the molecular level. These studies have revealed that the full-length p53 protein contains two independently folding domains: a sequence-specific DNA binding domain at the centre of the protein and a homo-tetramerization domain towards the C-terminus (Vogelstein et al, 2000). In addition, p53 contains three unstructured regions: an N-terminal transactivation domain, a linker between the DNA binding and oligomerization domains and a C-terminal basic region (Joerger and Fersht, 2008).

Several three-dimensional structures of the DNA binding domain of p53 have been determined both in the presence of specific DNA and in the absence of DNA (Cho et al, 1994; Ho et al, 2006; Malecka et al, 2009; Chen et al, 2010; Kitayner et al, 2006, 2010). All these structures encompass only the DNA binding domain. The most recent structures are derived from crystals containing four DNA binding domains in complex with specific DNA, thereby potentially recapitulating how full-length p53 tetramers recognize DNA (Malecka et al, 2009; Chen et al, 2010; Kitayner et al, 2010). With the exception of one structure, in which the p53 DNA binding domains had been chemically crosslinked to DNA (Malecka et al, 2009), the structures show that sequence-specific DNA binding is not accompanied by conformational changes within the p53 DNA binding domain (Cho et al, 1994; Ho et al, 2006; Chen et al, 2010; Kitayner et al, 2006, 2010). Yet, in the context of practically every other sequence-specific DNA binding protein characterized to date, the interaction with DNA is accompanied by conformational changes (Frankel and Kim, 1991; Alber, 1993; Spolar and Record, 1994). The significance of these conformational changes is not well understood, but their universal prevalence suggests that they may have an important role and raises the question why p53 is an exception.

The DNA binding domain of p53 is monomeric in solution and has micromolar affinity for DNA (Weinberg et al, 2005). Because of this, except for the crosslinked p53-DNA complex, all the other studied p53-DNA complexes assembled during crystallization, implying that crystal packing interactions have contributed to their formation and, hence, to their structure. In contrast, p53 polypeptides that encompass 
both the DNA binding and oligomerization domains have nanomolar affinity for sequence-specific DNA and form stable protein-DNA complexes in solution (Weinberg et al, 2005). We envisioned, therefore, that the three-dimensional structures of such complexes would be much less likely to be affected by crystal packing interactions.

We describe here structures of a multidomain p53 oligomer in the presence and absence of DNA. The structures reveal a conformational switch in loop L1, when p53 binds to specific DNA. Analysis of loop L1 mutants further shows that the conformational switch alters the kinetic properties of p53 DNA binding, allowing binding off-rates to be regulated independently of affinities. Since conformational switching is a characteristic of practically all sequence-specific DNA binding proteins (Frankel and Kim, 1991; Alber, 1993; Spolar and Record, 1994), our findings may be broadly relevant.

\section{Results}

\section{Crystallization of a thermostable multidomain p53 protein}

Our initial attempts to express p53 polypeptides that contained both the DNA binding and oligomerization domains in a soluble form were unsuccessful. To address this problem, we introduced stabilizing amino-acid substitutions in the DNA binding domain of human p53, which, in its wild-type form, has a very low melting temperature (Bullock et al, 1997). The designed substitutions targeted non-conserved residues away from the DNA binding surface and, generally, sampled residues from the repertoire present at that position in p53 proteins from other species (Soussi and May, 1996). The goal of these substitutions was to maximize the contribution of the hydrophobic effect to protein folding. One substitution, Arg209 to Pro, was designed computationally (Zhu et al, 2004). After many rounds of mutagenesis and functional testing, we obtained a stabilized (ST) human p53 DNA binding domain that had the same DNA binding specificity as wild-type p53 and was functional in cells (Figures 1 , 2A and B; Supplementary Figures S1-S6).

For crystallization, we expressed a p53 polypeptide lacking the unstructured $\mathrm{N}$-terminal and $\mathrm{C}$-terminal ends of the protein and most of the linker between the DNA binding and oligomerization domains. Further, two amino-acid substitutions were introduced in the tetramerization domain to convert it to a dimerization domain (Davison et al, 2001). Three p53 proteins modified in this way were studied; they are referred to as p53CR1, p53CR2 and p53CR3 and have deletions of 30,29 and 28 residues, respectively, in the linker between the DNA binding and oligomerization domains (Supplementary Figure S6).

The three-dimensional structure of p53CR1 in the absence of DNA was determined at a resolution of $2.1 \AA$ (Supplementary Table 1). As predicted, the protein crystallized as a dimer (Figure 2C). The stabilized DNA binding domains adopted the same conformation as the wild-type DNA binding domain (Cho et al, 1994). The dimerization domain also adopted the same conformation as the half wild-type tetramerization domain (Lee et al, 1994; Clore et al, 1995; Jeffrey et al, 1995; Davison et al, 2001). The DNA binding domains of the dimer did not interact with one another and it appears that their orientation relative to each other was dictated by crystal packing contacts.
A

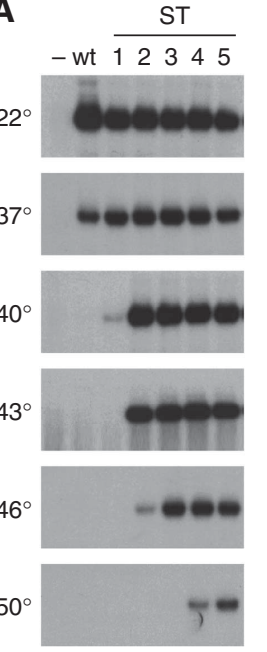

B

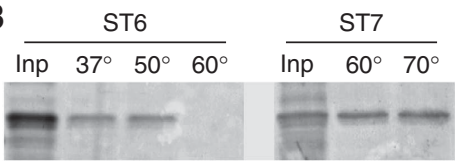

C

\begin{tabular}{c|cc|c} 
Codon & wt & ST7 & Species \\
\hline 135 & C & V & Squid \\
141 & C & V & None \\
146 & W & Y & None \\
182 & C & S & Hamster \\
203 & V & A & Hamster \\
209 & R & P & Clam \\
229 & C & Y & Mouse \\
233 & H & Y & None \\
234 & Y & F & Clam \\
235 & N & K & Mouse \\
236 & Y & F & Chicken \\
253 & T & V & None \\
268 & N & D & Mouse
\end{tabular}

Figure 1 Development of a stabilized human p53 protein. Stabilized proteins were developed via an iterative process of protein design and functional testing allowing the retention of substitutions that enhanced stability. About a hundred generations of stabilized proteins were generated, but, for brevity, only seven generations (ST1-ST7) are presented. The amino-acid substitutions present in the stabilized mutants are shown in Supplementary Figure S3. (A) Functional assay employed for the design of mutants ST1-ST5. Full-length wild-type human p53 and the stabilized mutants ST1-ST5 were translated in vitro using a rabbit reticulocyte lysate transcription/translation system (Promega Corporation). The proteins were then diluted in DNA binding buffer, heated at the indicated temperature for $10 \mathrm{~min}$, returned to room temperature and assayed for binding to $\left[{ }^{32} \mathrm{P}\right]$-labelled oligonucleotide $\mathrm{BC}$ (Supplementary Figure S1). (B) Assay employed for the design of mutants ST6-ST7. Proteins consisting of residues 94-358 of human p53 with an internal deletion of residues 292-321, containing stabilizing amino-acid substitutions in their DNA binding domains (ST6 or ST7), were expressed in E. coli. The soluble fraction of the $E$. coli lysate (Input, Inp) was heated for $10 \mathrm{~min}$ at the indicated temperature and the fraction that remained soluble was subjected to SDS-polyacrylamide gel electrophoresis and stained with Coomassie Brilliant Blue. (C) List of amino-acid substitutions, using the single letter amino-acid code, present in the DNA binding domain of the stabilized protein ST7 (hereafter referred to as ST).

The p53CR2 and p53CR3 proteins formed stable complexes with DNA oligonucleotides containing consensus p53 binding sites (Supplementary Figure S7), allowing their three-dimensional structures with DNA to be determined at resolutions of 2.4 and $3.2 \AA$, respectively (Supplementary Table 1 ). The two structures were essentially identical (Supplementary Figure S8) and so the description will focus on the higher resolution p53CR2-DNA structure.

The overall structure shows two p53CR2 dimers-having in total four DNA binding domains-bound to doublestranded DNA (Figure 2D and E). The DNA molecule contains four contiguous pentamer repeats, which we named a, $\mathrm{b}, \mathrm{c}$ and $\mathrm{d}$, reflecting their $5^{\prime}$ to $3^{\prime}$ order (Figure $2 \mathrm{~A}$ ). The major groove of each pentamer repeat is recognized by one p53 subunit, which bears the same label (A, B, C and D) as the repeat it contacts. The structure can recapitulate well how a full-length p53 tetramer would bind DNA. Unlike p53CR2, full-length p53 has long linkers between the DNA binding and oligomerization domains, allowing the oligomerization domains of all four subunits to interact with each other (Figure 2E). 
A

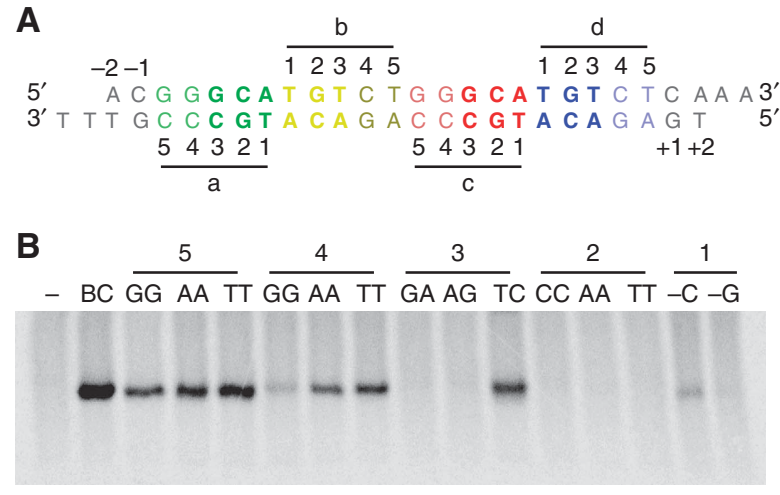

C

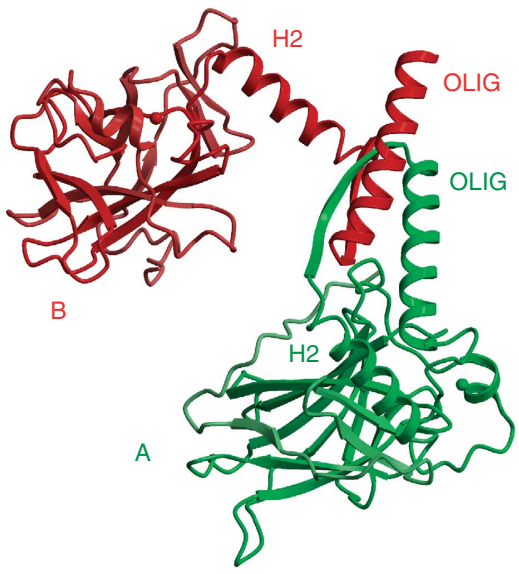

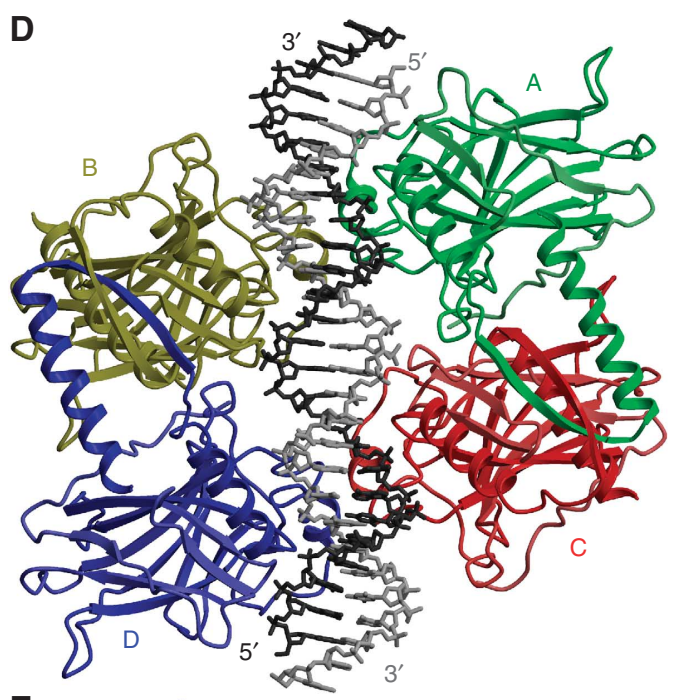

$\mathrm{E}$

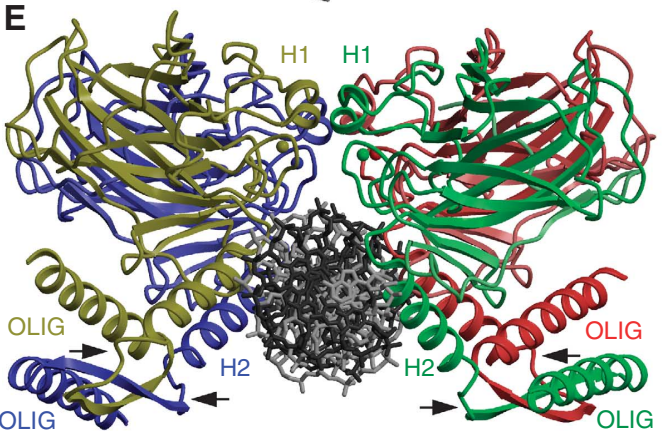

Figure 2 Overall three-dimensional structure of a multidomain p53 oligomer with and without bound DNA. (A) Sequence of the oligonucleotide, containing four contiguous pentamer repeats (a-d), that was crystallized in complex with DNA. (B) DNA binding specificity of the p53CR2 protein, as examined by an electrophoresis mobility shift assay using an oligonucleotide containing a high affinity binding site (BC; Supplementary Figure S1) and variants thereof that differ from BC by having the indicated bases at positions 1, 2, 3, 4 or 5 of the pentamer repeats. The first letter in the name of the variants refers to the base introduced in repeats a and $\mathrm{c}$ and the second letter to the base introduced in repeats b and d; - indicates that no substitution was made. (C) Overall three-dimensional structure of p53CR1 in the absence of DNA. The two subunits are labelled A and B. H2, helix 2 of the DNA binding domain; OLIG, oligomerization domain. (D) Overall three-dimensional structure of two p53CR2 dimers bound to DNA. The p53 subunits are labelled A-D. The DNA binding domain of subunit B has the same orientation as the DNA binding domain of subunit A of p53CR1 in panel (C). The DNA has complementary overhangs at each end and formed a pseudocontinuous double helix in the crystal. (E) The structure of the p53CR2-DNA complex rotated by $90^{\circ}$ relative to panel (D). The arrows indicate the linkers between the DNA binding and oligomerization domains.

\section{Validation of the structure}

While the overall structure of the p53CR2-DNA complex appears grossly similar to the recently described structures of four p53 DNA binding domains in complex with DNA (Malecka et al, 2009; Chen et al, 2010; Kitayner et al, 2010), none of the previous structures can be superimposed on the p53CR2-DNA structure or on each other (Supplementary Figure S9). One of the several differences between the various structures is the intersubunit interface between the DNA binding domains recognizing parallel non-consecutive pentamer repeats, for example, domains B and D. Two main areas of interaction are evident at this interface in the p53CR2-DNA structure (Figure $3 \mathrm{~A}$ and B). One area, near the bound DNA, is dominated by hydrogen bonds involving Thr123, Thr140, Glu198 and Gly199 from one subunit and Ser94, Ser96, Gln167 and Thr170 from the other subunit. The second area, away from the bound DNA, involves Val225, Gly226, Asp228 from one subunit and Ser99, Lys101, Tyr103, Leu264 and Arg267 from the other subunit.

This second area of interaction is unique to the p53CR2DNA structure among all the previously described p53 tetramer-DNA structures (Figure 3C; Supplementary Figure S9).
Gly226 appears critical for this interface, because glycine's small side chain permits the two subunits to come close to each other. We modelled the common rotamers of phenylalanine at this position. All these rotamers were incompatible with the p53CR2-DNA structure, as they overlapped with the side chains of either Arg267 or Tyr103 from the other subunit (Figure 3C and D). Yet, they were compatible with all the previously determined p53 tetramer structures, which are characterized by larger intersubunit distances (Figure 3C; Supplementary Figure S9).

As predicted by the p53CR2-DNA structure, the Gly226 to Phe substitution compromised the ability of full-length p53 to activate transcription from a binding site containing contiguous pentamer repeats and, importantly, substitution of Arg267 with serine acted as a second-site revertant (Figure 3E; Supplementary Figure S10). In contrast, the Gly226 to Phe substitution did not compromise the transcriptional activity of p53 from a DNA site with a 10 base pair insertion between pentamer repeats $b$ and c. This insertion moves p53 domains B and D away from each other and should make p53 tolerant to interface substitutions, as, indeed, was the case. Thus, second-site revertant mutagenesis 
A

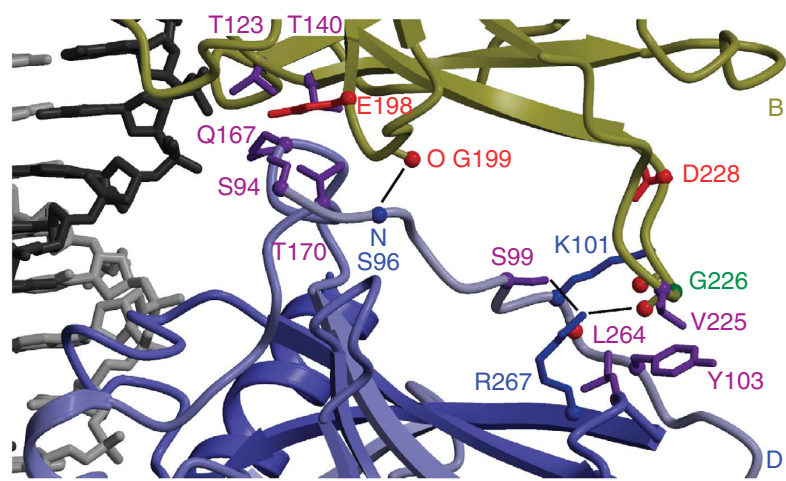

C

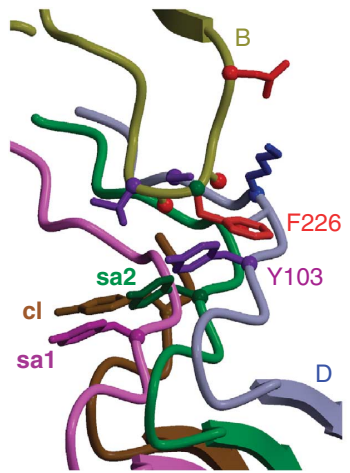

D

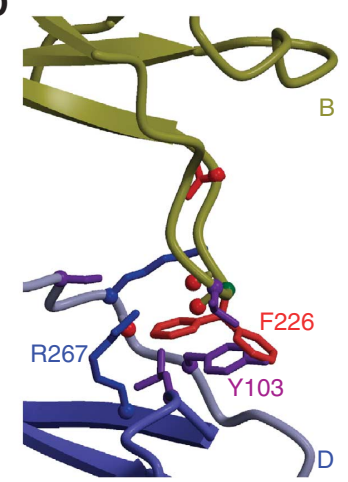

B

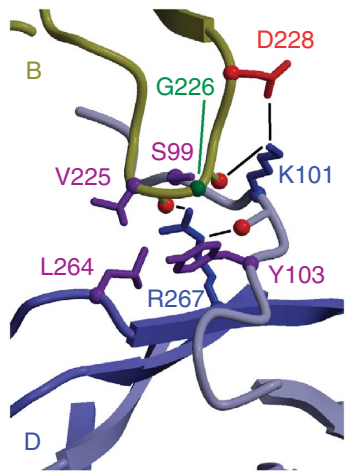

E

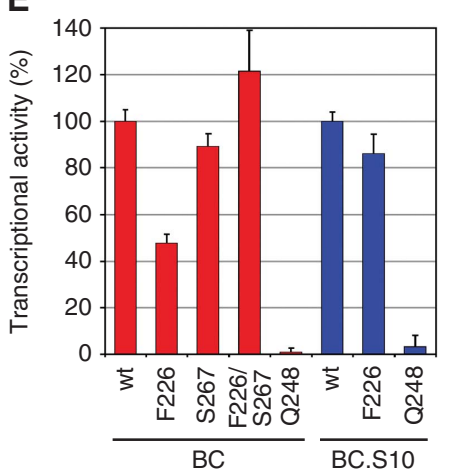

Figure 3 Functional analysis of the p53-p53 contacts at the parallel intersubunit interface. (A) Structure of the p53CR2-DNA complex rotated by $180^{\circ}$ relative to Figure $2 \mathrm{D}$. Only parts of subunits $\mathrm{B}$ and $\mathrm{D}$ are shown. The side chains of the residues that form the parallel intersubunit interface are indicated. (B) Structure of the p53CR2-DNA complex rotated by $90^{\circ}$ relative to panel (A). (C) Structure of the p53CR2-DNA complex showing only the N-terminal residues and first $\beta$-strand of the DNA binding domain of subunit D and the corresponding segments of the D subunits of the previously determined p53 tetramer structures (sa1, self-assembled 1, pdb 3KMD (Chen et al, 2010); sa2, self-assembled 2, pdb 3IGK (Kitayner et al, 2010); cl, crosslinked, pdb 3EXJ (Malecka et al, 2009)). The structures are superimposed on the basis of the B subunits and only the B subunit of the p53CR2-DNA complex is shown, for clarity. The side chain of Tyr103 (Y103) of all structures and the side chain of one possible phenylalanine rotamer at position 226 (F226) of subunit B are also shown. The orientation is the same as in panel (B). (D) Structure of the p53CR2-DNA complex showing the two other possible rotamers of phenylalanine at position 226 (F226) of subunit B. The orientation is the same as in panel (A). (E) Transcriptional activity of full-length p53 proteins containing the stabilized DNA binding domain and no additional substitutions (wt) or the following substitutions, as indicated: Gly226 to Phe (F226); Arg267 to Ser (S267); the double mutant (F226/S267); or the tumour-derived mutant Arg248 to Gln (Q248). Oligonucleotide BC has contiguous pentamer repeats; oligonucleotide BC.S10 has a 10 base pair insertion between repeats b and c (Supplementary Figure S1). Mean values and standard deviations from three independent experiments are shown. All p53 proteins were expressed at equal levels (Supplementary Figure S10).

analysis of the transcriptional activity of full-length p53 in human cells supports the p53CR2-DNA structure described here and not any one of the other previously determined p53 tetramer-DNA structures.

\section{DNA binding-induced conformational switch of loop L1} Contrary to the previously determined p53-DNA structures, we observed a conformational switch in loop L1 upon DNA binding. In the subunits contacting the inner repeats (repeats b and c; Figure 2A), loop L1 adopted an extended conformation that was similar to the conformation seen in the absence of DNA and in the previously determined self-assembled p53-DNA structures (Figure 4A; Supplementary Figure S11). In this conformation, Lys120 and Ser121 contacted the DNA. However, in the subunits contacting the outer repeats (repeats a and d), loop L1 adopted a recessed conformation and neither Lys120 nor Ser121 contacted the DNA; instead Arg283 from helix H2 made a DNA contact (Figure 4B). The recessed loop L1 conformation was similar to the one observed in the crosslinked p53 tetramer-DNA structure (Supplementary Figure S12; Malecka et al, 2009) and was also reminiscent of the loop L1 conformation observed in C. elegans p53 in the absence of DNA (Huyen et al, 2004).
In the crosslinked p53-DNA structure, the recessed conformation of loop L1 was present in all four subunits (Supplementary Figure S12) and was attributed to steric interference with the chemical crosslink (Malecka et al, 2009). However, in the p53-CR2 structure, the conformational switch of loop L1 was linked to a $3-\AA$ shift of the DNA helical axis at the centre of the p53 DNA binding site (Figure 4C; Supplementary Figure S13). As a result of this shift, the DNA backbone was displaced away from loop L1 of p53 subunits $B$ and $C$, providing space to accommodate the extended loop L1 in the major groove of the bound DNA (Figure 4D and E; Supplementary Figures S11 and S14). In contrast, at the edges of the p53 binding site, the DNA helical axis did not shift and loop L1 of subunits A and D adopted a recessed conformation to be able to accommodate to the standard B-form DNA (Figure 4D and E; Supplementary Figure S14).

\section{Effect of the loop L1 conformational switch on DNA binding kinetics}

The observed conformational changes in p53 and DNA in the p53CR2-DNA structure suggest that p53 binds DNA via an induced fit mechanism (Koshland, 1958). Induced fit, as 


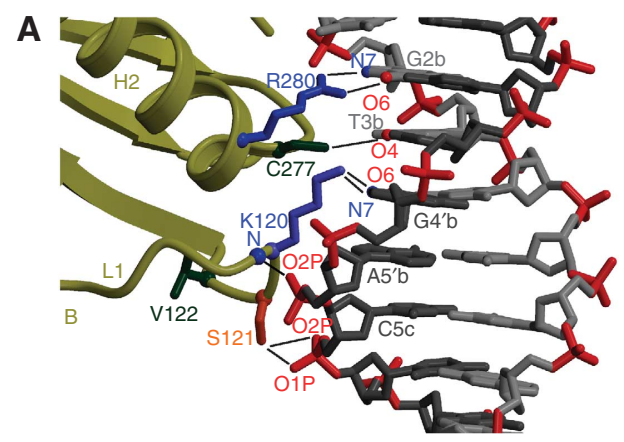

B

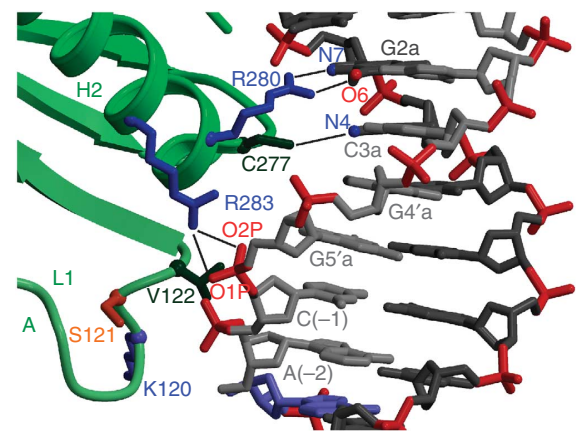

C

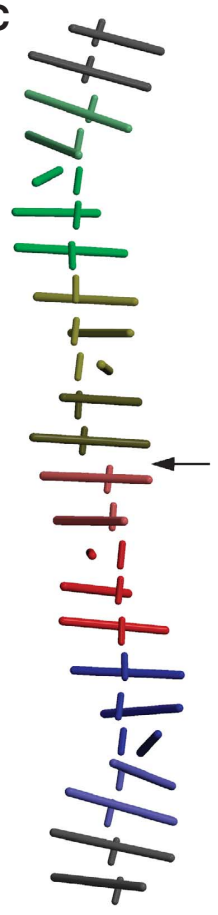

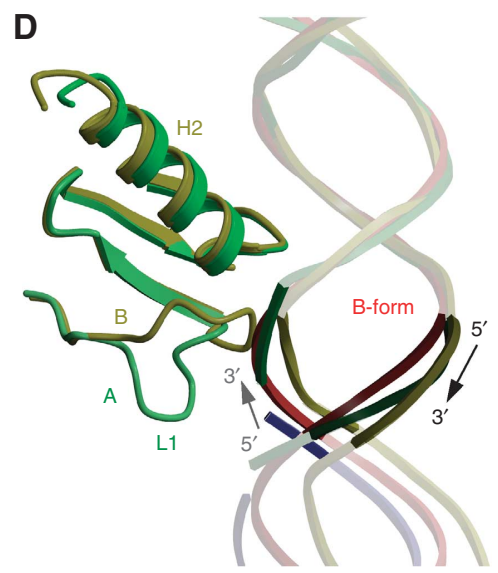

E

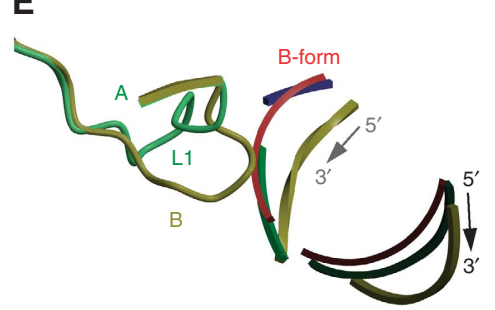

Figure 4 Conformational changes in p53 and DNA upon sequence-specific DNA binding. (A) The extended conformation of loop L1 of subunits B and C (subunit B is shown as an example). The contacts of residues Lys120 (K120), Ser121 (S121), Cys277 (C277) and Arg280 (R280) with DNA are shown. The side chain of Val122 (V122), which stabilizes the extended conformation of loop L1, is indicated. Bases are referred to by type (A, G, T, C), position in the pentamer repeats (1-5) and pentamer repeat labels (b, c), as shown in Figure 2A. H2, helix 2; L1, loop L1. (B) The recessed conformation of loop L1 of subunits A and D (subunit A is shown as an example). The contacts of residues Cys277 (C277), Arg280 (R280) and Arg283 (R283) with DNA are shown. The crystallographic symmetry-related DNA molecule at the bottom of the image is coloured blue. (C) $\mathrm{Cl}^{\prime}$ - $\mathrm{C1}^{\prime}$ vectors and helical axes for each base pair of the p53-bound DNA. All vectors are coloured according to the oligonucleotide sequence (as shown in Figure 2A). The orientation of the DNA is the same as in Figure 2D. The arrow indicates the DNA helical axis shift at the centre of the p53 binding site. (D) Comparison of the conformations of loop L1 of subunits A and B and of the adjacent DNA backbones. Subunit B and its neighbour DNA backbone are coloured yellow; subunit A and its neighbour DNA are coloured green; the crystallographic symmetry-related DNA next to subunit A is coloured blue. The backbone of an ideal B-form DNA is coloured red. The arrows indicate the $5^{\prime}$ to $3^{\prime}$ direction of each DNA strand. The orientation is the same as in panels (A), (B) and (C). (E) Same elements as shown in panel (D) viewed after a $90^{\circ}$ rotation. Only a slab is shown corresponding to the DNA segment with darker colours in panel (D). Helix H2 (not shown) would be above the plane of the paper.

compared with rigid body interactions, may not significantly affect binding affinities, but can have profound effects on binding kinetics (Pape et al, 1999; Johnson, 2008). To illustrate this, we first consider binding of p53 to DNA via a rigid body interaction. Under equilibrium conditions, the dissociation constant $K_{\mathrm{D}}$ is equal to the off-rate constant $k_{\text {off }}$ divided by the on-rate constant $k_{\text {on }}$ (Figure $5 \mathrm{~A}$ ). In the rigid body interaction model, the on-rate constant would be determined by diffusion and electrostatic attraction/repulsion and would be the same irrespective of the DNA sequence (von Hippel and Berg, 1989; Johnson, 2008). Thus, for different DNAs, the off-rate constant would be proportional to the $K_{\mathrm{D}}$, reflecting the fact that high affinity interactions are characterized by long half-lives (Figure 5A).

In the case of an induced fit mechanism, binding proceeds in two steps (Koshland, 1958). An initial interaction occurs without any conformational change, as described above for rigid body interactions, and then, in a second step, the initial interaction drives a conformational switch that allows a better fit between the protein and DNA (Figure 5B). The constant $k_{\text {on } 1}$ is determined by diffusion and electrostatic attraction/repulsion, whereas the constant $k_{\text {on } 2}$ is determined by the conformational switch (which may be different for different DNAs and for different p53 proteins; Johnson,
2008). If one considers macroscopic (overall) on-rate and off-rate constants, $k_{\text {ono }}$ and $k_{\text {offo, }}$, respectively, to describe both steps of the reaction, then the dissociation constant $K_{\mathrm{D}}$ equals $k_{\text {offo }}$ divided by $k_{\text {ono }}$ (Figure $5 \mathrm{~B}$ ). But because $k_{\text {ono }}$ depends partially on the conformational switch (which may be different for different DNAs and for different p53 proteins), the $k_{\text {offo }}$ values are not proportional to the $K_{\mathrm{D}}$ values. Therefore, one can distinguish between rigid body interaction and induced fit mechanisms by measuring $K_{\mathrm{D}}$ and $k_{\text {offo }}$ values for different p53-DNA complexes.

DNA binding affinities of p53 under equilibrium conditions have been previously studied by fluorescence anisotropy; at physiological salt concentrations the reported dissociation constants for high affinity sites were in the range of 1.1$4.2 \mathrm{nM}$, whereas non-specific DNAs were bound with affinities in the range of $29.8-88.6 \mathrm{nM}$ (Weinberg et al, 2004). The reported small differences in affinities for specific and non-specific DNAs is puzzling, since it makes it difficult to understand how human p53 identifies its specific target sites in a diploid genome containing six billion competing non-specific sites.

To study the DNA binding properties of p53, we also relied on fluorescence anisotropy. We employed proteins that span residues $79-393$ of human $\mathrm{p} 53$. These proteins contained the 
A

$$
\begin{aligned}
& \mathrm{p53}+\mathrm{DNA} \underset{k_{\text {off }}}{\stackrel{k_{\text {on }}}{\longrightarrow}} \mathrm{p53/DNA} \\
& K_{\mathrm{D}}=\frac{[\mathrm{p} 53][\mathrm{DNA}]}{[\mathrm{p} 53 / \mathrm{DNA}]}=\frac{k_{\text {off }}}{k_{\text {on }}}
\end{aligned}
$$

C

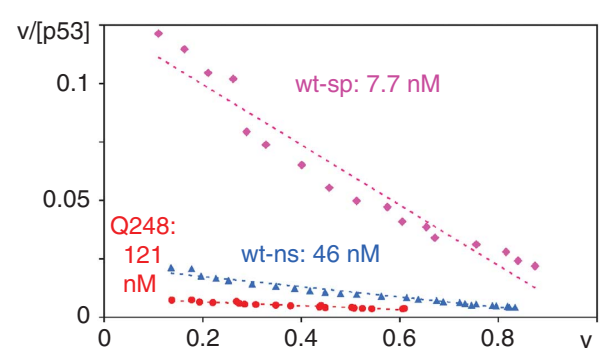

E

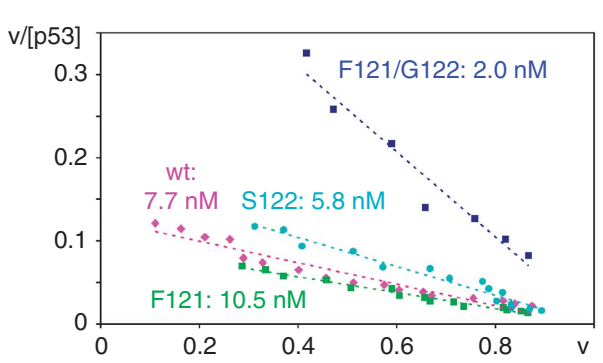

B

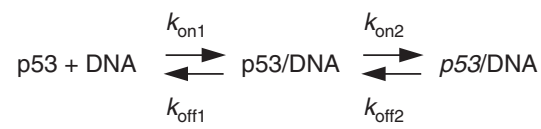

$K_{\mathrm{D}}=\frac{[\mathrm{p} 53][\mathrm{DNA}]}{[p 53 / \mathrm{DNA}]}=\frac{k_{\text {offo }}}{k_{\text {ono }}}$

D

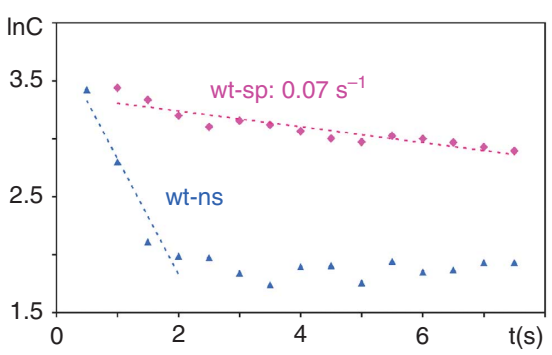

$\mathbf{F}$

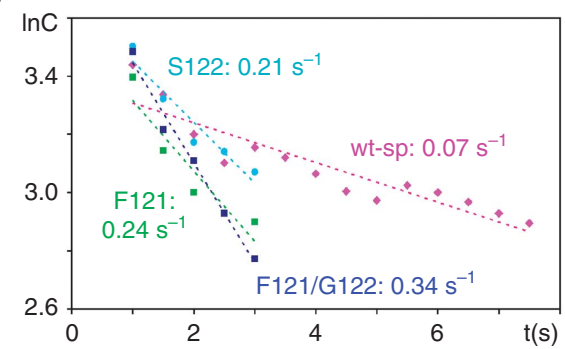

Figure 5 Loop L1 regulates p53 DNA binding off-rates. (A) Equations describing the formation of p53-DNA complexes via a rigid body

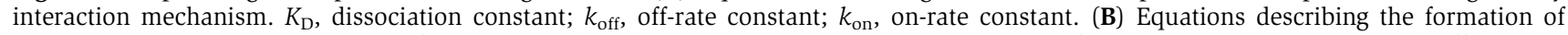
p53-DNA complexes via an induced fit mechanism. p53 (in italics) indicates the DNA-bound conformation. $k_{\text {offo }}$ and $k_{\text {ono, overall off-rate and }}$ on-rate constants, respectively. (C) Scatchard plots for binding of stabilized wild-type p53 (residues 79-393) to specific (sp) DNA (oligonucleotide ABC.4TT, Supplementary Figure S1) and non-specific (ns) DNA (oligonucleotide NS, Supplementary Figure S1) and of the stabilized tumour-derived mutant Gln284 (Q248) to specific DNA. The $K_{\mathrm{D}}$ values are indicated in nM. The slope of the fitted lines is equal to $-1 / K_{\mathrm{D}} \cdot v=[\mathrm{p} 53 / \mathrm{DNA}] /([\mathrm{p} 53 / \mathrm{DNA}]+[\mathrm{DNA}])$. (D) Plot of the natural logarithm of the concentration (lnC) of the complexes of wild-type p53 with fluorescent-specific (sp) and non-specific (ns) DNA versus time (in s). Excess non-fluorescent competitor DNA was added at time zero. The $k_{\text {off }}$ values, which are equal to the slope of the fitted lines, are indicated in $\mathrm{s}^{-1}$. (E) Scatchard plots for binding of stabilized wild-type and loop L1 p53 mutants (residues 79-393) to specific (sp) DNA (oligonucleotide ABC.4TT). F121, Phe121; S122, Ser122; F121/G122, Phe121/ Gly122 double mutant. The $K_{\mathrm{D}}$ values are indicated in nM. (F) Plot of the natural logarithm of the concentration (lnC) of the complexes of wildtype 553 and p53 loop L1 mutants with fluorescent-specific DNA versus time (in s). Excess non-fluorescent competitor DNA was added at time zero. The $k_{\text {off }}$ values are indicated in $\mathrm{s}^{-1}$.

stabilized DNA binding domain (to prevent denaturation during the experiment). However, unlike the proteins used for crystallization, the proteins used for the DNA binding studies contained a wild-type tetramerization domain, an intact, non-truncated, linker between the DNA binding and oligomerization domains and an intact C-terminus. Thus, these proteins contained all regions of p53 known to regulate DNA binding (Hupp et al, 1992; Muller-Tiemann et al, 1998).

By Scatchard plot analysis, the macroscopic dissociation constants for a specific DNA and for a non-specific DNA were 7.7 and $46 \mathrm{nM}$, respectively, only a six-fold difference (Figure 5C). Using the same assay, the tumour-derived p53 mutant $\mathrm{Gln} 248$ had a $K_{\mathrm{D}}$ value for specific DNA of $121 \mathrm{nM}$, arguing that the wild-type p53 values reflected binding of the p53 DNA binding domain to DNA and not other non-specific interactions. The small difference in affinities for specific and non-specific DNAs suggests that affinity on its own is unlikely to be sufficient for p53 to recognize its specific targets in the genome.

To measure off-rate constants, the p53 protein was bound to fluorescent DNA; then 20-fold excess of non-fluorescent DNA of the same sequence was added, while fluorescence anisotropy measurements were being acquired in kinetic mode. Displacement of specific fluorescent DNA from the wild-type p53 protein by excess specific non-fluorescent DNA proceeded over about $30 \mathrm{~s}$ (Supplementary Figure S15A), allowing us to calculate an off-rate constant of $0.07 \mathrm{~s}^{-1}$ (Figure 5D). In contrast, displacement of non-specific fluorescent DNA by excess non-specific non-fluorescent DNA was completed in $<1 \mathrm{~s}$, which is the time it took for the reagents to mix (our own unpublished observations), making it impossible to determine a precise off-rate value (Figure $5 \mathrm{D}$; Supplementary Figure S15A). Nevertheless, we can conclude that the difference in off-rates for specific and non-specific DNAs is greater than the difference in affinities.

The p53CR2-DNA structure shows that both p53 and DNA switch conformation upon sequence-specific binding. In p53, the major conformational change in the DNA binding domain involves loop L1. Therefore, one would expect that aminoacid substitutions that facilitate the conformational switch of loop L1 would lower the kinetic barrier for sequence-specific DNA binding and this would be reflected by higher off-rate constants. In the absence of DNA, the extended conformation of loop L1 is stabilized by Val122, which forms a small 
hydrophobic core at the centre of the loop (Figure 4A). In the recessed conformation, the hydrophobic side chain of Val122 has flipped towards the solvent (Figure 4B), which is energetically unfavourable and, therefore, possibly part of the kinetic barrier for the conformational switch. Thus, substitution of Val122 with serine or glycine should facilitate the conformational switch.

Ser121 faces towards solvent in the extended loop L1 conformation, but in the recessed conformation it faces towards the centre of loop L1 (Figure 4A and B). Substitution of Ser121 with phenylalanine enhances the affinity of p53 for DNAs that contain one p53 half-site, that is, a site with only two specific pentamer repeats; whereas, this same substitution has varied and sequence-dependent effects on the affinity of p53 for DNAs that contain a fulllength p53 site (Saller et al, 1999; Kakudo et al, 2005; Zupnick and Prives, 2006). We modelled the rotamers of phenylalanine at position 121 in the context of the recessed loop L1 conformation. Interestingly, the most common rotamer places the phenylalanine hydrophobic side chain towards the centre of loop L1 (Supplementary Figure S16), raising the possibility that a Ser121 to Phe substitution would also facilitate the conformational switch between the extended and recessed conformations.

Based on the above considerations, we generated three mutants targeting loop L1: Ser121 to Phe, Val122 to Ser and the double mutant Ser121 to Phe and Val122 to Gly and determined their dissociation and off-rate constants. The single mutants had affinities for specific DNA very similar to those of wild-type p53, whereas the double mutant had a four-fold higher affinity for specific DNA than wild-type p53 (Figure 5E). This would be consistent with the latter mutant using a lower fraction of its binding energy for the conformational switch. Yet, despite having equal or higher affinities for specific DNA than wild-type p53, all three loop L1 mutants had higher off-rates constants (Figure 5F; Supplementary Figure S15B). Of significance, the Phe121-Gly122 double mutant, which had a four-fold higher affinity for specific DNA than wild-type p53, formed complexes with DNA, whose half-life was five times shorter than those of wildtype p53. These results suggest that the conformational switch of loop L1 allows DNA binding off-rates to be regulated independently of affinities.

Consistent with a low off-rate value (i.e., a long half-life of the p53-DNA complex) being important for p53 function in cells, one study reported that substitution of Val122 with glycine abolished transcriptional activity (Kakudo et al, 2005). In our hands, all loop L1 mutants maintained transcriptional activity, but at reduced levels compared with wildtype p53 (Supplementary Figure S17). Some residual activity is not surprising. These mutants have higher off-rates than wild-type p53, but not as high as tumour-derived p53 mutants (Supplementary Figure S15). Further, the affinity of some loop L1 mutants for specific DNA was several-fold higher than that of wild-type p53 (Figure 5E).

\section{Discussion}

Several structures of p53 with DNA have been previously determined (Cho et al, 1994; Ho et al, 2006; Malecka et al, 2009; Chen et al, 2010; Kitayner et al, 2006, 2010). An unusual feature of all these structures, with the exception of one structure of p53 crosslinked with DNA (Malecka et al, 2009), is that neither the DNA binding domain of p53, nor the DNA undergo major conformational changes upon sequence-specific DNA binding. In contrast, conformational changes are a characteristic of practically every other sequence-specific DNA binding protein characterized to date (Frankel and Kim, 1991; Alber, 1993; Spolar and Record, 1994), raising the question why p53 would be an exception. The p53-DNA structure reported here is the first structure of a p53 polypeptide that includes both the DNA binding domain and the oligomerization domain and the first structure to exhibit a conformational switch upon sequencespecific DNA binding.

We propose that the structure reported here is the physiologically relevant structure of a p53 tetramer-DNA complex. First, the presence of the oligomerization domain allows p53 to form stable complexes with DNA in solution (Supplementary Figure S7). Therefore, p53-DNA complex formation preceded crystallization. In contrast, isolated p53 DNA binding domains bind DNA weakly (Weinberg et al, 2005) and only form complexes with DNA during crystallization (Cho et al, 1994; Chen et al, 2010; Kitayner et al, 2006, 2010); therefore, the assembly of these complexes is guided both by p53-DNA contacts and by crystal packing contacts. In this regard, it is not surprising that the previously reported p53 tetramer-DNA structures all differ from one another (Supplementary Figure S9). Second, the structure reported here is insensitive to variations in the linker length between the p53 DNA binding and oligomerization domains and insensitive to variations in the oligonucleotide used. While we had to truncate the linker between the DNA binding and oligomerization domains, a common practice in crystallography to reduce conformational flexibility that inhibits crystallization, two p53 polypeptides with different size linkers, p53CR2 and p53CR3, formed essentially identical structures (Supplementary Figure S8). Thus, linker length variation did not impact on how the four DNA binding domains interacted with DNA. We also obtained crystals of p53CR2 and p53CR3 with oligonucleotides containing different specific DNA sites and/or different overhangs ( $5^{\prime}$ versus $3^{\prime}$ and different overhang sequences) and in every case the structures were identical to the one reported here (our own unpublished observations). Thus, so far, we have observed one unique p53 tetramer-DNA conformation. Third, we validated our structure by second-site revertant mutagenesis using fulllength p53 proteins expressed in human cells (Figure 3).

The main question that arises from our structure relates to the significance of the conformational switch targeting loop L1. Interestingly, it appears that the propensity of loop L1 to adopt a recessed conformation is conserved in evolution, since the recessed conformation, observed here in human p53, is practically identical to the recessed conformation observed in all four subunits of mouse p53 crosslinked to DNA (Supplementary Figure S12; Malecka et al, 2009) and similar to the conformation of loop L1 of $C$. elegans p53 (Huyen et al, 2004). Further, Lys120, at the tip of loop L1, is acetylated in response to DNA damage and this acetylation, which has the potential to affect the conformation of the loop, regulates p53 DNA binding specificity in vivo (Sykes et al, 2006). As discussed below, our experiments suggest that the conformational switch of loop L1 is critical for the ability of p53 to distinguish specific from non-specific DNA. 
It has been proposed that sequence-specific DNA binding proteins are able to recognize their specific sites in the genome because their affinities for specific and non-specific DNAs differ by 3-7 orders of magnitude (Spolar and Record, 1994). However, in many studies in the past, DNA binding affinities were measured under non-equilibrium conditions by filter binding and electrophoretic mobility shift assays (e.g., Klig et al, 1987; Hellman and Fried, 2007). Because complexes of proteins with non-specific DNA, typically have half-lives in the order of milliseconds (von Hippel and Berg, 1989; Elf et al, 2007), such complexes dissociate in nonequilibrium assays, leading to underestimation of their affinities. When p53 DNA binding was studied under equilibrium conditions and at physiological salt concentrations (Weinberg et al, 2004 and this study), the difference in affinities for specific and non-specific DNAs was $<10$-fold, which makes it very difficult to explain how p53 recognizes its target sites in the genome. We propose that the conformational switch of loop L1 addresses this problem by allowing DNA binding off-rates to be regulated independently of affinities and that it is the large difference in binding offrates that distinguishes specific from non-specific DNA sites.

To our knowledge, a model, whereby sequence-specific DNA binding proteins modulate DNA binding off-rates independently of affinities in order to discriminate specific targets from non-specific DNA, has not been previously proposed (Kalodimos et al, 2004a). Yet, this model may help explain the almost universal prevalence of conformational switches in protein-DNA interactions (Frankel and Kim, 1991; Alber, 1993; Spolar and Record, 1994). One can envision that, at any given time, most molecules of a sequence-specific DNA binding protein would be bound to nonspecific DNA, because of the relatively high affinity for such DNA. However, the very high off-rate for non-specific DNA means that the protein molecules would rapidly bounce from DNA site to DNA site, via sliding and intersegment transfer, as previously proposed (von Hippel and Berg, 1989; McKinney et al, 2004). When a specific DNA site is identified, the protein and/or the DNA would switch conformation, resulting in a significant decrease in the on-rate and off-rate constants-akin to locking the protein at the specific DNA site-without a major increase in affinity. In support of this model, it is noteworthy that proteins other than p53 also have high affinity for non-specific DNA at physiological salt concentrations (Oda et al, 1998) and that binding of sequencespecific proteins to non-specific DNA is not accompanied by conformational switching (Winkler et al, 1993; Newman et al, 1995; Lewis et al, 1996; Viadiu and Aggarwal, 2000; Kalodimos et al, 2004b).

\section{Materials and methods}

\section{Protein sample preparation and crystallization}

p53 polypeptides were expressed in $E$. coli. The cells were lysed in buffer consisting of $25 \mathrm{mM}$ bis-tris propane (BTP); ( $\mathrm{pH} 6.8$ ), $250 \mathrm{mM} \mathrm{NaCl}, 5 \mathrm{mM}$ DTT and protease inhibitors, and the polypeptides were purified by cation exchange (Sepharose SP column; Pharmacia Biotech, Uppsala, Sweden) and gel filtration (Superdex 200 column; Pharmacia Biotech) chromatography. After purification, the p53 protein was concentrated to $8 \mathrm{mg} \mathrm{ml}^{-1}$ in $25 \mathrm{mM}$ BTP (pH 6.8), $150 \mathrm{mM} \mathrm{NaCl}, 5 \mathrm{mM}$ DTT buffer and was crystallized under standard hanging drop vapour diffusion conditions in 48-well plates (Hampton Research, Aliso Viejo, CA, USA) or in a novel microfluidics system (Emamzadah et al, 2009) at $4^{\circ} \mathrm{C}$ in the absence of DNA or in the presence of DNA at a 1:1.1 protein:DNA molar ratio. Complexes of p53 with oligonucleotides 22-28 base pairs long were examined for crystallization, after being isolated by gel filtration chromatography. Crystals were obtained only with 26 base pair long oligonucleotides. The precipitant solutions used for crystallization were $0.2 \mathrm{M}$ ammonium acetate, $0.1 \mathrm{M}$ HEPES ( $\mathrm{pH}$ 7.5), 45\% v/v 2-Methyl-2,4-pentanediol for p53CR1 in the absence of DNA and 0.15 M DL-Malic Acid (pH 7.0), $20 \%$ Polyethylene Glycol 3350 for the p53CR2-DNA and p53CR3DNA complexes.

\section{Data collection and structure refinement}

The p53CR1 and p53CR2 data sets were collected at the ID23-2 microfocus beamline of the European Synchrotron Radiation Facility (Grenoble, France); the p53CR3 data sets were collected at the ID14-1 beamline of the same facility. Reflection data were indexed, integrated and scaled using the CCP4 software package. Initial solutions were determined by molecular replacement and refined under non-crystallographic symmetry restraints using the programs $\mathrm{CNS}$ and $\mathrm{O}$. Base pair origin vectors were determined using the program 3DNA.

\section{Fluorescence anisotropy}

The p53 proteins were expressed in $E$. coli and purified to homogeneity, as described above. For determination of dissociation constants, fluorescein-labelled oligonucleotides were diluted to a concentration of $23 \mathrm{nM}$ in buffer consisting of $25 \mathrm{mM} \mathrm{BTP}$ (pH 6.8), $150 \mathrm{mM} \mathrm{NaCl}, 2 \mathrm{mM}$ DTT and $1 \%$ Tween-20; $2 \mathrm{ml}$ of this solution were placed in a quartz cuvette and anisotropy measurements were obtained with a fluorescence spectrophotometer (Cary Eclipse) that was custom fitted with emission and excitation polarizer filters; p53 protein was then titrated by adding small amounts in increments until the anisotropy values reached a plateau. Typically, the p53 protein concentration was titrated from 1 to $100 \mathrm{nM}$ and each titration curve corresponded to about 25 data points. Measurements were initiated $30 \mathrm{~s}$ after each addition of $\mathrm{p} 53$ protein to ensure that the reaction had proceeded to equilibrium. Excitation and emission wavelengths were set at 490 and $516.4 \mathrm{~nm}$, respectively, and the averaging time for each measurement was $40 \mathrm{~s}$. The anisotropy data were used to generate Scatchard plots without applying 'correction' factors. The Scatchard plots were then used to calculate dissociation constants. For both specific and non-specific DNAs, we considered that one p53 tetramer could bind one double-stranded DNA molecule, because the short length of the oligonucleotides precludes binding of two tetramers. Thus, our calculations refer to macroscopic, rather than to microscopic, dissociation constants.

For determination of off-rate constants, the same set-up was used. Anisotropy values were determined with fluorescein-labelled oligonucleotide alone $(69 \mathrm{nM})$ and after addition of $50 \mathrm{nM}$ p53 protein. Then the spectrophotometer was operated in kinetic mode acquiring emission spectra with both polarizers at the vertical position and an averaging time of $0.5 \mathrm{~s}$; during this time a 20 -fold excess of non-fluorescent DNA of the same sequence as the fluorescent DNA was added and manually mixed for $1 \mathrm{~s}$. After stabilization of the values, anisotropy measurements were obtained. Offrate constants were determined by applying equations for first-order kinetic reactions, which were found to fit the data well. All anisotropy experiments were performed at room temperature.

\section{Electrophoretic mobility gel shift assay}

$\left[{ }^{35} \mathrm{~S}\right]$-methionine-labelled p53 proteins were expressed by in vitro translation in rabbit reticulocyte lysate (Promega Corporation, Madison, WI, USA) and assayed for DNA binding, as previously described (Waterman et al, 1995; Huyen et al, 2004).

\section{Tumour suppression and transactivation assays}

Tumour suppressor and transcriptional activities were assayed in Saos-2 p53-/- osteosarcoma cells, as previously described (Waterman et al, 1996).

\section{Accession numbers}

Three-dimensional structure coordinates and structure factors have been deposited at the Protein Data Bank under the accession numbers 3Q01, 3Q05 and 3Q06. 


\section{Supplementary data}

Supplementary data are available at The EMBO Journal Online (http://www.embojournal.org).

\section{Acknowledgements}

We thank Nikola Pavletich, Philip Jeffrey and Gregory van Duyne for initial guidance on crystallization of p53-DNA complexes; Ueli Schibler, Uli Laemmli and Jules Shafer for helpful discussions; Jennifer Waterman, Stephanie Cafarella, Ivy Loreto, Kimberly Harris, Emily Sheston, Sarah Sterman and Laurence Tropia for technical help; the staff of the ESRF, Grenoble, France for help in collecting the X-ray diffraction data; the Swiss National Foundation (TDH and EV), the National Institutes of Health, USA (TDH and JGS) and the European Commission Project GENICA (TDH) for funding.

\section{References}

Alber T (1993) Protein-DNA interactions: how GCN4 binds DNA. Curr Biol 3: 182-184

Bartkova J, Horejsi Z, Koed K, Kramer A, Tort F, Zieger K, Guldberg P, Sehested M, Nesland JM, Lukas C, Orntoft T, Lukas J, Bartek J (2005) DNA damage response as a candidate anti-cancer barrier in early human tumorigenesis. Nature 434: $864-870$

Bullock AN, Henckel J, DeDecker BS, Johnson CM, Nikolova PV, Proctor MR, Lane DP, Fersht AR (1997) Thermodynamic stability of wild-type and mutant p53 core domain. Proc Natl Acad Sci USA 94: $14338-14342$

Chen Y, Dey R, Chen L (2010) Crystal structure of the p53 core domain bound to a full consensus site as a self-assembled tetramer. Structure 18: 246-256

Cho Y, Gorina S, Jeffrey PD, Pavletich NP (1994) Crystal structure of a p53 tumor suppressor-DNA complex: understanding tumorigenic mutations. Science 265: 346-355

Clore GM, Ernst J, Clubb R, Omichinski JG, Kennedy WM, Sakaguchi K, Appella E, Gronenborn AM (1995) Refined solution structure of the oligomerization domain of the tumour suppressor p53. Nat Struct Biol 2: 321-333

Davison TS, Nie X, Ma W, Lin Y, Kay C, Benchimol S, Arrowsmith $\mathrm{CH}$ (2001) Structure and functionality of a designed p53 dimer. J Mol Biol 307: 605-617

Elf J, Li GW, Xie XS (2007) Probing transcription factor dynamics at the single-molecule level in a living cell. Science 316: 1191-1194

Emamzadah S, Petty TJ, De Almeida V, Nishimura T, Joly J, Ferrer JL, Halazonetis TD (2009) Cyclic olefin homopolymer-based microfluidics for protein crystallization and in situ X-ray diffraction. Acta Crystallogr D Biol Crystallogr 65: 913-920

Frankel AD, Kim PS (1991) Modular structure of transcription factors: implications for gene regulation. Cell 65: 717-719

Gorgoulis VG, Vassiliou LV, Karakaidos P, Zacharatos P, Kotsinas A, Liloglou T, Venere M, Ditullio Jr RA, Kastrinakis NG, Levy B, Kletsas D, Yoneta A, Herlyn M, Kittas C, Halazonetis TD (2005) Activation of the DNA damage checkpoint and genomic instability in human precancerous lesions. Nature 434: 907-913

Halazonetis TD, Gorgoulis VG, Bartek J (2008) An oncogeneinduced DNA damage model for cancer development. Science 319: $1352-1355$

Hellman LM, Fried MG (2007) Electrophoretic mobility shift assay (EMSA) for detecting protein-nucleic acid interactions. Nat Protoc 2: $1849-1861$

Ho WC, Fitzgerald MX, Marmorstein R (2006) Structure of the p53 core domain dimer bound to DNA. J Biol Chem 281: 20494-20502

Hollstein M, Sidransky D, Vogelstein B, Harris CC (1991) p53 mutations in human cancers. Science 253: 49-53

Hupp TR, Meek DW, Midgley CA, Lane DP (1992) Regulation of the specific DNA binding function of p53. Cell 71: 875-886

Huyen Y, Jeffrey PD, Derry WB, Rothman JH, Pavletich NP, Stavridi ES, Halazonetis TD (2004) Structural differences in the DNA binding domains of human p53 and its C. elegans ortholog Cep1. Structure 12: 1237-1243

Jeffrey PD, Gorina S, Pavletich NP (1995) Crystal structure of the tetramerization domain of the p53 tumor suppressor at 1.7 angstroms. Science 267: 1498-1502
Author contributions: The overall study was conceived and designed by TDH with important contributions from all other authors. TDH designed all the substitutions, except for Pro209, which was designed by JGS and TJP. The crystals were obtained by TJP, SE and ESS; the X-ray diffraction data were collected and processed by TJP, SE and TDH; the fluorescence anisotropy experiments were performed and analysed by SE, IP, EV and TDH; the electrophoretic mobility shift assays and tissue culture experiments were performed by LC and TJP. TDH wrote the manuscript with the help of all the authors.

\section{Conflict of interest}

The authors declare that they have no conflict of interest.

Joerger AC, Fersht AR (2008) Structural biology of the tumor suppressor p53. Annu Rev Biochem 77: 557-582

Johnson KA (2008) Role of induced fit in enzyme specificity: a molecular forward/reverse switch. J Biol Chem 283: 26297-26301

Kakudo Y, Shibata H, Otsuka K, Kato S, Ishioka C (2005) Lack of correlation between p53-dependent transcriptional activity and the ability to induce apoptosis among 179 mutant p53s. Cancer Res 65: 2108-2114

Kalodimos CG, Boelens R, Kaptein R (2004a) Toward an integrated model of protein-DNA recognition as inferred from NMR studies on the Lac repressor system. Chem Rev 104: 3567-3586

Kalodimos CG, Biris N, Bonvin AM, Levandoski MM, Guennuegues M, Boelens R, Kaptein R (2004b) Structure and flexibility adaptation in nonspecific and specific protein-DNA complexes. Science 305: $386-389$

Kan Z, Jaiswal BS, Stinson J, Janakiraman V, Bhatt D, Stern HM, Yue P, Haverty PM, Bourgon R, Zheng J, Moorhead M, Chaudhuri S, Tomsho LP, Peters BA, Pujara K, Cordes S, Davis DP, Carlton VE, Yuan W, Li L et al. (2010) Diverse somatic mutation patterns and pathway alterations in human cancers. Nature 466: 869-873

Kastan MB, Onyekwere O, Sidransky D, Vogelstein B, Craig RW (1991) Participation of p53 protein in the cellular response to DNA damage. Cancer Res 51: 6304-6311

Kern SE, Kinzler KW, Bruskin A, Jarosz D, Friedman P, Prives C, Vogelstein B (1991) Identification of p53 as a sequence-specific DNA-binding protein. Science 252: 1708-1711

Klig LS, Crawford IP, Yanofsky C (1987) Analysis of trp repressor-operator interaction by filter binding. Nucleic Acids Res 15: 5339-5351

Kitayner M, Rozenberg H, Kessler N, Rabinovich D, Shaulov L, Haran TE, Shakked Z (2006) Structural basis of DNA recognition by p53 tetramers. Mol Cell 22: 741-753

Kitayner M, Rozenberg H, Rohs R, Suad O, Rabinovich D, Honig B, Shakked Z (2010) Diversity in DNA recognition by p53 revealed by crystal structures with Hoogsteen base pairs. Nat Struct Mol Biol 17: 423-429

Koshland Jr DE (1958) Application of a theory of enzyme specificity to protein synthesis. Proc Natl Acad Sci USA 44: 98-104

Kuerbitz SJ, Plunkett BS, Walsh WV, Kastan MB (1992) Wild-type p53 is a cell cycle checkpoint determinant following irradiation. Proc Natl Acad Sci USA 89: 7491-7495

Lee W, Harvey TS, Yin Y, Yau P, Litchfield D, Arrowsmith CH (1994) Solution structure of the tetrameric minimum transforming domain of p53. Nat Struct Biol 1: 877-890

Lewis M, Chang G, Horton NC, Kercher MA, Pace HC, Schumacher MA, Brennan RG, Lu P (1996) Crystal structure of the lactose operon repressor and its complexes with DNA and inducer. Science 271: 1247-1254

Malecka KA, Ho WC, Marmorstein R (2009) Crystal structure of a p53 core tetramer bound to DNA. Oncogene 28: 325-333

McKinney K, Mattia M, Gottifredi V, Prives, C (2004) p53 linear diffusion along DNA requires its C terminus. Mol Cell 16: 413-424

Muller-Tiemann BF, Halazonetis TD, Elting JJ (1998) Identification of an additional negative regulatory region for p53 sequencespecific DNA binding. Proc Natl Acad Sci USA 95: 6079-6084 
Newman M, Strzelecka T, Dorner LF, Schildkraut I, Aggarwal AK (1995) Structure of Bam HI endonuclease bound to DNA: partial folding and unfolding on DNA binding. Science 269: 656-663

Oda M, Furukawa K, Ogata K, Sarai A, Nakamura H (1998) Thermodynamics of specific and non-specific DNA binding by the c-Myb DNA-binding domain. J Mol Biol 276: 571-590

Pape T, Wintermeyer W, Rodnina M (1999) Induced fit in initial selection and proofreading of aminoacyl-tRNA on the ribosome. EMBO J 18: 3800-3807

Saller E, Tom E, Brunori M, Otter M, Estreicher A, Mack DH, Iggo R (1999) Increased apoptosis induction by $121 \mathrm{~F}$ mutant p53. EMBO J 18: 4424-4437

Soussi T, May P (1996) Structural aspects of the p53 protein in relation to gene evolution: a second look. J Mol Biol 260: 623-637

Spolar RS, Record Jr MT (1994) Coupling of local folding to sitespecific binding of proteins to DNA. Science 263: 777-784

Sykes SM, Mellert HS, Holbert MA, Li K, Marmorstein R, Lane WS, McMahon SB (2006) Acetylation of the p53 DNA-binding domain regulates apoptosis induction. $\mathrm{Mol}$ Cell 24: 841-851

Viadiu H, Aggarwal AK (2000) Structure of BamHI bound to nonspecific DNA: a model for DNA sliding. Mol Cell 5: 889-895

Vogelstein B, Lane D, Levine AJ (2000) Surfing the p53 network. Nature 408: $307-310$ von Hippel PH, Berg OG (1989) Facilitated target location in biological systems. J Biol Chem 264: 675-678

Waterman JL, Shenk JL, Halazonetis TD (1995) The dihedral symmetry of the p53 tetramerization domain mandates a conformational switch upon DNA binding. EMBO J 14: 512-519

Waterman MJ, Waterman JL, Halazonetis TD (1996) An engineered four-stranded coiled coil substitutes for the tetramerization domain of wild-type p53 and alleviates transdominant inhibition by tumor-derived p53 mutants. Cancer Res 56: 158-163

Weinberg RL, Veprintsev DB, Fersht AR (2004) Cooperative binding of tetrameric p53 to DNA. J Mol Biol 341: 1145-1159

Weinberg RL, Veprintsev DB, Bycroft M, Fersht AR (2005) Comparative binding of $\mathrm{p} 53$ to its promoter and DNA recognition elements. J Mol Biol 348: 589-596

Winkler FK, Banner DW, Oefner C, Tsernoglou D, Brown RS, Heathman SP, Bryan RK, Martin PD, Petratos K, Wilson KS (1993) The crystal structure of EcoRV endonuclease and of its complexes with cognate and non-cognate DNA fragments. EMBO J 12: 1781-1795

Zhu YJ, Fu XR, Wang T, Tamura A, Takada S, Saven JG, Gai F (2004) Guiding the search for a protein's maximum rate of folding. Chem Phys 307: 99-109

Zupnick A, Prives C (2006) Mutational analysis of the p53 core domain L1 loop. J Biol Chem 281: 20464-20473 